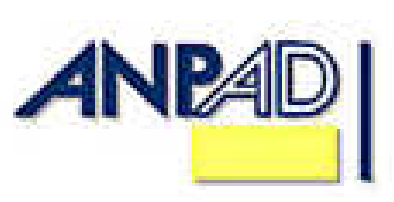

Disponível em

http://www.anpad.org.br/rac

RAC, Curitiba, v. 15, n. 6, art. 5,

pp. 1059-1077, Nov./Dez. 2011

$\left(\right.$ (c) EY-NO $^{-1}$

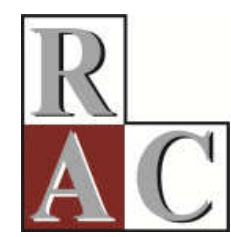

\title{
Relações Quantitativas entre Orientação para o Mercado, Desempenho Organizacional e a Percepção Externa de Sucesso em Instituições de Pesquisas Tecnológicas Afiliadas à ABIPTI
}

\author{
Quantitative Relationships between Market Orientation, Organizational Performance \\ and External Perception of Success or Failure in Technological Research Institutions \\ Affiliated to ABIPTI
}

Newton Amaral Paim*
E-mail: npaim@cdtn.br
Centro de Desenvolvimento da Tecnologia Nuclear - CDTN
Belo Horizonte, MG, Brasil.
José Edson Lara
E-mail: jedson2010@ hotmail.com
Faculdades Pedro Leopoldo - UNIPEL
Pedro Leopoldo, MG, Brasil.
Juliana Maria Magalhães Christino
E-mail: julianam.prof@ gmail.com
Belo Horizonte, MG, Brasil.

* Endereço: Newton Amaral Paim

Av. Presidente Antônio Carlos, 6627, Campus da UFMG, Belo Horizonte/MG, 31270-901.

Copyright (C) 2011 RAC. Todos os direitos, até mesmo de tradução, são reservados. É permitido citar parte de artigos sem autorização prévia, desde que seja identificada a fonte. 


\title{
Resumo
}

O tema orientação para o mercado vem ocupando posição central na teoria do marketing nas últimas décadas. A maioria dos estudos empíricos nessa área baseia-se na realidade de países industrializados e no setor empresarial. A pesquisa empírica sobre o tema voltada para as organizações que não visam necessariamente ao lucro continua limitada. Este estudo investigou a relação da orientação para o mercado com o desempenho organizacional e com a percepção externa de sucesso ou insucesso nas atividades-fim em instituições de pesquisas tecnológicas no Brasil, afiliadas à Associação Brasileira das Instituições de Pesquisas Tecnológicas (ABIPTI). Usando técnicas estatísticas uni e multivariadas, validaram-se os construtos em relação às formas de aferição utilizadas, a saber: indicadores de desempenho organizacional do prêmio de gestão ABIPTI; Escala MARKOR adaptada e validada para os propósitos do estudo e Painel de Especialistas, enquete aplicada em um conjunto de conhecedores do setor de ciência e tecnologia brasileiro. Como resultado, obtiveram-se bons índices de ajustamento para a estrutura teórica proposta e confirmaram-se todas as quatro hipóteses e três hipóteses derivadas de pesquisa testadas. Os resultados da pesquisa demonstraram que a orientação para o mercado das instituições de pesquisas tecnológicas estudadas mantém uma relação positiva e significativa com o desempenho organizacional das mesmas instituições; tal associação é nitidamente perceptível por um conjunto de especialistas no setor de Ciência \& Tecnologia brasileiro e externo àquelas instituições, ao avaliarem quão bemsucedidas, ou não tão bem-sucedidas, são as mesmas instituições no desempenho de suas atividades-fim.

Palavras-chave: Ciência \& Tecnologia (C\&T); escala MARKOR; indicadores de desempenho organizacional; orientação para o mercado; painel de especialistas.

\begin{abstract}
The theme of Market Orientation has occupied a central position in the theory of marketing in recent decades. The main empirical studies in this field have focused on industrialized countries and the managerial sector. Empirical research in the market orientation field focusing on the type of organization that does not necessarily pursue profit remains limited. In an attempt to examine the importance of market orientation, this study investigates its relationship with organizational performance and external perceptions of success or failure in the core activities of Brazilian technological research institutions affiliated with the Brazilian Association of Technological Research Institutions (ABIPTI). Univariate and multivariate statistical techniques were used to validate constructs concerning the measurement methods employed, namely: indicators of organizational performance of the ABIPTI management prize; the MARKOR scale adapted and validated for the purposes of the study and Panel of Experts, applied to a set of experts in the sector of science and technology in Brazil. The results show high levels of adjustment to the proposed theoretical structure, and confirm all four hypotheses and the three research-derived hypotheses that were testedtested. The results of the study show that market orientation in the technological research institutions under study has a positive and significant relationship with the organizational performance of these institutions, and this association is clearly visible to a group of experts in the Brazilian science and technology field who are external to these institutions, in assessing how successful or unsuccessful these institutions are in carrying out their core activities.
\end{abstract}

Key words: science and technology (S\&T); MARKOR scale; organizational performance indicators; market orientation; specialist panel. 


\section{Introdução}

O tema orientação para o mercado vem despertando grande interesse desde a década de 1990 e assumiu um lugar de destaque, tanto teórico quanto prático, no âmbito do marketing. Embora exista na literatura especializada uma série de estudos empíricos sobre o assunto, uma revisão, levada a efeito entre 2009 e 2010 pelos autores deste artigo, revelou que a maioria deles se baseia no ambiente dos países industrializados e/ou em organizações que visam ao lucro, isto é, em fabricantes de produtos ou em prestadoras de serviços. A pesquisa empírica sobre orientação para o mercado continua limitada, tanto em países desenvolvidos, quanto nos chamados periféricos, especialmente no Brasil e, em particular, analisando organizações que não visam diretamente ao lucro.

O conceito clássico de orientação para o mercado provém do trabalho seminal de Kohli e Jaworski (1990, p. 6), que o definiram, literalmente, nos seguintes termos: "a disposição de uma organização em coletar, disseminar e utilizar as informações dos clientes para responder rapidamente a oportunidades de mercado ou mudar as necessidades dos clientes". A proeminência do prestígio desse conceito advém do final da década de 1980 e início da década de 1990, quando as discussões teóricas sobre o marketing se direcionaram para a construção de arcabouços conceituais integrativos, sendo frequentes as discussões sobre as possíveis fontes de vantagens competitivas e sustentáveis. A partir de então, a crença dominante passou a ser de que a orientação para o mercado incrementava as possibilidades de obtenção de alto grau de desempenho nos negócios, apoiada no fato de que a almejada vantagem competitiva requereria equilíbrio entre as perspectivas do cliente e as da concorrência.

A qualidade do desempenho, por outro lado, apresentada pelas organizações envolvidas na disputa por um determinado mercado, é estudada há muito mais tempo; por exemplo, por Drucker (1954), e sua importância adquire contornos dramáticos, quando se trata de um setor sujeito a turbulências, ou com uma alta taxa de inovações, como é o caso de instituições de pesquisas tecnológicas. Esse autor propunha que as empresas mais inovativas tendem a alcançar melhores desempenhos que, por sua vez, são frequentemente associados em muitos estudos empíricos, à orientação para o mercado daquelas organizações.

Finalmente, a importância da percepção externa de sucesso ou insucesso corporativo baseia-se metaforicamente no dito popular (e milenar) que afirma: "Não basta, à mulher de César, ser virtuosa, mas tem de parecer virtuosa"; ou seja, de que adianta uma organização apresentar bons indicadores de desempenho e ser orientada para o mercado, se isto não é percebido pela sociedade ou, pelo menos, por um segmento importante e especializado desta?

Na tentativa, portanto, de contribuir para ampliar a compreensão do tema, o presente estudo objetivou investigar uma relação, supostamente positiva, entre três conceitos: o primeiro, de orientação para o mercado; o segundo, de desempenho organizacional; e um terceiro que aferiu a percepção externa de sucesso ou insucesso corporativo de um conjunto de instituições de pesquisas tecnológicas brasileiras, públicas e privadas, por meio de uma consulta a um grupo de especialistas no setor e externo àquele conjunto de instituições, a seguir denominado, no contexto deste trabalho, de Painel de Especialistas. Considerou-se que as instituições de pesquisas tecnológicas afiliadas à Associação Brasileira de Instituições de Pesquisas Tecnológicas (ABIPTI) representavam de maneira adequada esta população.

Por que a percepção externa de sucesso ou insucesso corporativo, ainda que de natureza subjetiva, é importante? A resposta se consubstancia no fato de que a exposição de uma imagem bemsucedida de uma instituição de pesquisas tecnológicas à sociedade, mesmo que resultado parcial de uma boa reputação pregressa, contribui para convalidar a relação positiva entre orientação para o mercado e desempenho organizacional, sendo, ao mesmo tempo, retro-alimentada por esses dois fatores num processo contínuo, circular e cíclico, conforme será defendido ao longo do presente texto. 


\section{O Problema de Pesquisa}

Em termos dos impactos e desafios desse tema para a sociedade contemporânea e, em especial, para a realidade brasileira, no âmbito do sistema nacional de fomento ao desenvolvimento científico e tecnológico, de acordo com as principais conclusões da pesquisa de Souza e Sbragia (2002), os Institutos Tecnológicos Industriais (ITI) são organizações extremamente complexas. Sua clientela é de natureza multifacetada e requer serviços diferenciados, daí a necessidade de uma constante atenção à realidade de seu entorno; seus objetivos institucionais precisam ser constantemente revisados, para se adequarem às alterações sofridas no macroambiente. Cabe, portanto, aos ITI conduzir a maior parte de suas ações orientadas para o mercado, uma vez que lhes compete assumir uma parcela dos riscos inerentes às próprias atividades industriais. Orientar-se, porém, somente pela demanda (ou seja, para os sinais emitidos pelo mercado) não significa necessariamente a autossustentação; sempre existe a necessidade de um aporte de recursos financeiros por parte de um, ou mais, mantenedores públicos; daí a existência de instrumentos de fomento a essas atividades de Pesquisa e Desenvolvimento (P\&D) e também de uma parcela de recursos provenientes da prestação de serviços e da venda de produtos tecnológicos ao setor privado. Essa é a problemática que o presente artigo pretende ajudar a elucidar com seus achados empíricos, a seguir resumida pelas seguintes perguntas de pesquisa:

1. As instituições de pesquisas tecnológicas brasileiras com maiores escores de orientação para o mercado apresentam simultaneamente maiores escores de desempenho organizacional do que as suas contrapartes com menores escores de orientação para o mercado?

2. As instituições de pesquisas tecnológicas brasileiras podem ser classificadas em um continuum de sucesso ou insucesso corporativo perceptível por um conjunto de especialistas no setor e externo àquelas instituições?

\section{Objetivo Geral}

Comprovar a existência de uma associação positiva, estatisticamente significativa e simultânea entre os somatórios dos escores de orientação para o mercado, de desempenho organizacional e de sucesso ou insucesso corporativo em instituições de pesquisas tecnológicas brasileiras.

\section{Objetivos Específicos}

1. Analisar e adaptar os critérios de desempenho organizacional para o âmbito das instituições de pesquisas tecnológicas brasileiras, a partir dos indicadores de desempenho da Associação Brasileira de Instituições de Pesquisas Tecnológicas (ABIPTI).

2. Analisar e adaptar os critérios de orientação para o mercado, segundo a Escala MARKOR, desenvolvida por Kohli, Jaworski e Kumar (1993), validada para o idioma português por Sampaio (2000) e adaptada para o âmbito das instituições de pesquisas tecnológicas brasileiras.

3. Identificar a presença de escores diferenciados de sucesso ou insucesso corporativo para as instituições de pesquisas tecnológicas brasileiras, obtidos por meio da percepção de um grupo de especialistas externos àquele conjunto de instituições, com base em sua isenção, experiência e conhecimento sobre o setor.

4. Comprovar a existência de uma associação positiva e estatisticamente significativa entre os somatórios dos escores de orientação para o mercado e de desempenho organizacional das instituições de pesquisas tecnológicas brasileiras. 
5. Comprovar a existência de uma associação positiva e estatisticamente significativa entre os somatórios dos escores de orientação para o mercado e de sucesso corporativo para o mesmo conjunto de instituições de pesquisas tecnológicas brasileiras.

6. Comprovar a existência de uma associação positiva e estatisticamente significativa entre os somatórios dos escores de desempenho organizacional e de sucesso corporativo para o mesmo conjunto de instituições de pesquisas tecnológicas brasileiras.

\section{Referencial Teórico}

A relação entre orientação para o mercado e desempenho foi colocada na pauta dos estudos sobre o tema e, a partir dos trabalhos pioneiros de Kohli e Jaworski (1990) e de Narver e Slater (1990), distintas versões e novas possibilidades surgiram, tendo ocupado a agenda de discussões teóricas sobre tal relação. Na grande maioria dessas propostas, a relação entre orientação para o mercado e desempenho encontrada é frequente e comprovadamente positiva (Appiah-Adu, 1998; Avlonitis \& Gounaris; 1997; Baker \& Sinkula, 1999; Becker \& Homburg, 1999; Caruana, Ramaseshan, \& Ewing, 1996; Didonet, 2007; Egeren \& O’Connor, 1998; Horng \& Chen, 1998; Kumar, Subramanian, \& Yauger, 1997; Narver \& Slater, 1990; Pelham, 1997; Pelham \& Wilson, 1996; Pitt, Caruana, \& Berthon, 1996; Raju, Lonial, \& Gupta, 1995; Slater \& Narver, 1994). Nessas investigações, os resultados revelaram que a orientação para o mercado proporciona, com raras exceções, melhorias no desempenho da empresa. Porém, ainda que um vasto campo de conhecimento tenha sido produzido nas últimas décadas sobre orientação para o mercado e sobre a sua relação com o desempenho, alguns setores de atividade têm recebido pouca atenção para o estudo do tema, como o de organizações que não visam necessariamente ao lucro e, particularmente, o de instituições públicas e privadas de pesquisas tecnológicas, em que os estudos sobre a implementação da orientação para o mercado permanecem praticamente inexistentes. Em termos empíricos, portanto, a presente pesquisa é relevante em relação ao segmento a ser estudado - o setor de pesquisas tecnológicas subsidiadas pelo governo ou pela iniciativa privada - que não tem recebido praticamente nenhuma atenção em termos de estudos empíricos sobre a orientação para o mercado.

Entre as escassas referências encontradas na literatura, cujos subsídios puderam ser adaptados e aproveitados para o presente trabalho, pode-se citar a pesquisa de Cervera, Mollá e Sánchez (2001), que estudou os antecedentes e consequências da orientação para o mercado em organizações públicas na Espanha, e o trabalho de Wood, Bhuian e Kiecker (2000) que avaliou o grau de orientação para o mercado em organizações hospitalares que não visavam ao lucro nos Estados Unidos.

Uma pesquisa cujo desenho se aproxima daquilo que se pretendeu empreender neste trabalho é a de Gupta, Bhojwani, Koshal e Koshal (2000). Segundo esses autores, a interação com o usuário é o mais importante ingrediente para o alcance de uma orientação para o mercado; as percepções de diretores e de cientistas seniores lotados em instituições de pesquisas tecnológicas, publicamente subsidiadas na Índia, foram utilizadas para se avaliar o fator interação com a indústria. As áreas examinadas incluíram: importância e frequência da interação das instituições de pesquisas tecnológicas com a indústria; barreiras enfrentadas pelas instituições de pesquisas tecnológicas em seus esforços para interagirem com a indústria e iniciativas tomadas pelas instituições de pesquisas tecnológicas para aperfeiçoarem sua interação com a indústria.

\section{O enfoque de Kohli e Jaworski sobre orientação para o mercado}

Ao reconhecerem a ampla importância dada à orientação ao mercado e admitirem as lacunas existentes na literatura, Kohli e Jaworski (1990, p. 1) justificaram a necessidade de "prover uma definição operacional, desenvolver proposições e construir um arcabouço compreensivo para orientar futuras pesquisas". Assim, esses autores partiram para a definição de seu próprio construto, 
fundamentando-o na literatura sobre marketing, em outras disciplinas e em pesquisas de campo realizadas com executivos alocados em diversas funções, níveis hierárquicos e organizações.

No contraste entre a visão dos executivos e a literatura, Kohli e Jaworski (1990) encontraram evidências para sugerir que o conceito de orientação para o mercado consiste em três aspectos fundamentais: geração de inteligência; disseminação de inteligência e responsividade. A definição formal seria: "orientação para o mercado é a ampla geração de inteligência de mercado concernente às necessidades dos atuais e futuros clientes, disseminação da inteligência através dos departamentos e a proatividade na resposta dada a elas" (Kohli \& Jaworski, 1990, p. 6). Após definirem o seu próprio conceito de orientação para o mercado e de proporem um modelo teórico com seus fatores antecedentes e consequentes, Kohli et al. (1993) desenvolveram uma medida de orientação para o mercado: a Escala MARKOR.

\section{Métricas para aferir o desempenho empresarial / organizacional}

A sobrevivência, o crescimento e, porque não mencionar, a boa reputação e a imagem positiva no desempenho das atividades-fim dependem das habilidades dos administradores em reconhecerem os desafios organizacionais que enfrentam, desvendarem novas oportunidades e escolherem a resposta administrativa correta para promoverem as mudanças internas necessárias para o cumprimento da missão previamente formulada para determinada organização. A forma consagrada de incentivo à avaliação qualitativa contínua do desempenho organizacional é a promoção periódica de premiações às boas práticas de gestão, conferidas por meio da inspeção in loco por parte de avaliadores credenciados nas organizações que se candidatam aos prêmios e também pela institucionalização do registro de indicadores de desempenho organizacional, cuja comparação, tanto entre diferentes pessoas-jurídicas, quanto ao longo do tempo em uma mesma organização, atestando o sucesso ou insucesso na implementação de práticas de gestão que validem (ou não) o bom desempenho organizacional e reforcem (ou não) a imagem de competência para determinada entidade.

Tanto Ghirlanda (2001) quanto Ferreira (2003) e Hourneaux, Correa e Maximiano (2006) defendem que os Prêmios de Qualidade são instrumentos apropriados para avaliar, tanto a gestão de organizações públicas, quanto a administração daquelas que representam os interesses privados. Por meio desse tipo de premiação é possível medir os níveis de gestão das organizações em relação ao estado da arte preconizado. A sistemática utilizada para avaliar as organizações em prêmios de qualidade consiste, em geral, na descrição do método e da aplicação das práticas de gestão, na apresentação dos resultados operacionais e nas conclusões relatadas, culminando com a comparação dos indicadores de desempenho demonstrados pelas organizações concorrentes, de acordo com as metas estabelecidas voltadas para o cumprimento da missão institucional, até se chegar a um vencedor, ou seja, a organização que superou seus competidores naquela disputa.

No âmbito específico das organizações de cunho empresarial, cujos parâmetros de aferição do desempenho privilegiam métricas diferentes daquelas adotadas pelas organizações que não visam diretamente ao lucro, tem sido verificado, nas proposições teóricas sobre orientação para o mercado, em especial, nos relatos sobre os estudos realizados sobre o tema, que várias são as medidas de desempenho adotadas pelos pesquisadores, para explicitarem os resultados auferidos pelas empresas em termos de desempenho e, em seguida, neles verificar o impacto dos níveis de orientação para o mercado delas. Amplamente citadas em estudos que tratam do desempenho empresarial, principalmente em investigações sobre estratégia, tais métricas podem ser agrupadas, de acordo com Venkatraman e Ramanujam (1986), em três grandes domínios: (a) desempenho financeiro; (b) desempenho financeiro e operacional; (c) efetividade, ou em medidas adotadas por diversos outros autores, que combinem o desempenho financeiro com o mercadológico, tais como lucratividade, crescimento em vendas e ganhos em participação de mercado entre outras.

No sentido de endossar a escolha e a definição dos parâmetros limitadores da presente pesquisa, cujo relato dos seus achados se avizinha, isto é, tanto a explanação, quanto a argumentação anteriormente apresentadas neste artigo indicam que, em se tratando de um setor que não visa 
diretamente ao lucro, as medidas de desempenho empresarial utilizadas naqueles estudos relatados não teriam nenhum sentido, tanto é que se preferiu rebatizá-lo, no presente caso, como desempenho organizacional; para medir e comparar as performances dos diversos institutos de pesquisas tecnológicas, foram empregados os indicadores de desempenho especialmente disponibilizados pela ABIPTI para este trabalho.

O Projeto Excelência na Pesquisa Tecnológica da ABIPTI foi criado em maio de 1998, a partir de uma reunião realizada pela ABIPTI; seus institutos de pesquisas tecnológicas associados e a Fundação Nacional da Qualidade (FNQ), de onde provieram as fórmulas de cálculo para a construção dos indicadores, inspiradas nos parâmetros de avaliação do Prêmio Nacional da Qualidade (PNQ) desta Fundação que, por sua vez, inspiraram-se no modelo norte-americano do Prêmio Nacional de Qualidade Malcolm Baldrige, conferido anualmente pelo National Institute of Standards and Technology (NIST). O objetivo geral do Projeto Excelência é promover a melhoria no desempenho dos IPTs, visando torná-lo mais competitivo. Nesse sentido, o processo de avaliação no âmbito do projeto deve representar para os IPTs um instrumento de gestão para o aprimoramento de suas atividades de pesquisa, desenvolvimento e serviços.

\section{O sucesso corporativo}

Segundo Miller e Friesen (1978, p. 924), o conceito de sucesso corporativo encontra-se relacionado com o "grau em que as organizações são capazes de atingir seus objetivos, apesar de sujeitas às restrições de viabilidade no longo prazo". Uma noção adjacente é a de autoperpetuação organizacional formulada por Chandler (1977), ou seja, a capacidade de a empresa sobreviver a seus próprios membros. De acordo com o pano de fundo teórico pesquisado por Fleck (2009), o sucesso organizacional é um dos temas centrais, em se tratando de estudos gerenciais; o crescimento é frequentemente citado como indicador adequado para aferi-lo. Conforme observado por Whetten (1980), o crescimento organizacional é uma hipótese implícita em estudos acadêmicos sobre o tema sucesso nos negócios, porque geralmente se assume a existência de uma correlação positiva entre tamanho e idade nas organizações estudadas, embora outras definições de sucesso enfatizem mais a dimensão temporal. Bem menos popular, entretanto, que a noção de sucesso organizacional mencionada por Whetten $(1980,1987)$, o fracasso também é citado na literatura sob diferentes formas, que incluem a "morte ou mortalidade organizacional", saída do negócio, falência, diminuição de tamanho (downsizing) e declínio (Mellahi \& Wilkinson, 2004, p. 22).

Em seu estudo sobre centros públicos de pesquisas cooperativas entre indústrias e universidades nos EUA, Geisler, Furino e Kiresuck (1990) identificaram como medidas operacionais de sucesso, em ordem decrescente de importância atribuída pelos respondentes pesquisados: a viabilidade e a continuidade de existência do centro de pesquisas; a estabilidade financeira; a taxa de transferência de tecnologia à indústria; a reputação do centro de pesquisas; as realizações da universidade; a taxa de retenção de estudantes; a taxa de retenção de membros provenientes da indústria e o arranjo eficaz e sistemático da liderança do centro de pesquisas. Em outro trabalho acadêmico, Fritz (1996) também investigou o sucesso corporativo, definido por esse autor como o grau em que as metas de competitividade; de satisfação dos clientes; de garantia de continuidade da organização e de lucratividade de longo prazo seriam atingidas, valendo-se, para tal, de uma amostra probabilística de cento e quarenta e quatro firmas industriais da Alemanha Ocidental. Os vários testes de validade e de confiabilidade empreendidos sustentaram estatisticamente, de acordo com o autor, a robustez e a pertinência do modelo baseado naqueles quatro critérios como medida central para o sucesso corporativo.

\section{A percepção como medida avaliadora do sucesso corporativo}

A proposta de utilizar a percepção como medida alternativa para avaliar o sucesso corporativo pode caracterizar-se como representação mais próxima da realidade, tratando-se do desempenho organizacional dos institutos de pesquisas tecnológicas na relação com sua orientação para o mercado. Isso porque as medidas de inputs e de outputs utilizadas estarão diretamente relacionadas à qualidade 
de atendimento prestado aos clientes por esse tipo de organização, que não visa diretamente ao lucro e à sua preocupação em atender as necessidades destes. Assim, esse aporte teórico adotado traz a proposição de uma medida de desempenho que engloba e proporciona mais visibilidade aos fatores específicos inerentes ao setor investigado.

No caso do presente estudo, com o objetivo de obter uma avaliação coerente - já que o interesse foi tentar determinar o aspecto sucesso corporativo no desempenho das atividades finalísticas em instituições públicas de pesquisas tecnológicas - foi utilizada a percepção como forma de medida e, como outputs, a opinião agregada e o julgamento em um painel composto por indivíduos altamente qualificados e experientes, constituído por professores e pesquisadores ligados ao estudo da C\&T, cientistas de renome, representantes de órgãos governamentais de fomento a $\mathrm{C} \& \mathrm{~T}$, diretores e técnicos de associações de classe ligadas a $\mathrm{C} \& \mathrm{~T}$ e por dirigentes e técnicos da área de $\mathrm{C} \& \mathrm{~T}$ de empresas tecnologicamente intensivas e, eventualmente, clientes dos produtos e serviços tecnológicos oferecidos pelas instituições de pesquisas estudadas.

A utilização do fator percepção para resolver problemas, ou para avaliar e prever determinados eventos, é relativamente consagrada, quando se necessita compreender e captar a opinião majoritária em matérias de natureza controvertida e complexa: capacidade de prever o sucesso, ou o fracasso, em projetos de desenvolvimento de novos produtos ou processos por parte de seus próprios responsáveis (Souder, 1969); percepção sobre iniciativa inovadora (Forehand, 1963); técnica DELPHI (Tersine \& Riggs, 1976); classificação de inovações em maiores ou menores na indústria de instrumentos científicos e eletrônicos (Hippel, 1977); determinação do grau de inovatividade em escritórios de arquitetura (Blau \& Mckinley, 1979); e também por Paim (1984), para medir o grau de inovatividade em empresas brasileiras montadoras de produtos de informática.

Após essas considerações, o sucesso ou insucesso corporativo, no contexto do presente trabalho, fica, finalmente, assim definido como:

O sucesso ou insucesso corporativo das instituições de pesquisas tecnológicas brasileiras referese ao fato de elas, independentemente da área de conhecimento em que atuem, apresentarem ao público especializado externo um desempenho perceptivelmente diferenciado (superior, ou inferior) em suas atividades-fim, isto é, aquelas diretamente relacionadas com o desenvolvimento de estudos que permitam a criação e o oferecimento contínuo de produtos e serviços tecnológicos que atendam aos propósitos do governo, do mercado e da sociedade em geral.

\section{As Hipóteses e Hipóteses Derivadas do Estudo}

H1: Existe uma convergência significativa entre os somatórios dos escores de orientação para o mercado, de desempenho organizacional e de percepção externa de sucesso ou insucesso corporativo nas instituições de pesquisas tecnológicas brasileiras associadas à ABIPTI.

H2: Existe uma correlação positiva e significativa entre os somatórios dos escores de orientação para o mercado e de desempenho organizacional nas instituições de pesquisas tecnológicas brasileiras associadas à ABIPTI.

Partindo-se da hipótese $\mathbf{H 2}$ e respeitando-se a natureza tripartite do construto empregado para definir orientação para o mercado, que foi a escala MARKOR, composta por geração de inteligência, disseminação de inteligência e responsividade (Kohli, Jaworski, \& Kumar, 1993), estabelecem-se três hipóteses derivadas relacionadas com a segunda hipótese do estudo.

H2a: Existe uma correlação positiva e significativa entre os somatórios dos escores de geração de inteligência na orientação para o mercado e de desempenho organizacional nas instituições de pesquisas tecnológicas brasileiras associadas à ABIPTI. 
H2b: Existe uma correlação positiva e significativa entre os somatórios dos escores de disseminação inteligência na orientação para o mercado e de desempenho organizacional nas instituições de pesquisas tecnológicas brasileiras associadas à ABIPTI.

H2c: Existe uma correlação positiva e significativa entre os somatórios dos escores de responsividade na orientação para o mercado e de desempenho organizacional nas instituições de pesquisas tecnológicas brasileiras associadas à ABIPTI.

H3: Existe uma correlação positiva e significativa entre os somatórios dos escores de orientação para o mercado e de percepção externa de sucesso ou insucesso corporativo nas instituições de pesquisas tecnológicas brasileiras associadas à ABIPTI.

H4: Existe uma correlação positiva e significativa entre os somatórios dos escores de desempenho organizacional e de percepção externa de sucesso ou insucesso corporativo nas instituições de pesquisas tecnológicas brasileiras associadas à ABIPTI.

\section{Metodologia}

A pesquisa desenvolvida foi do tipo descritivo-conclusiva e a metodologia, centrada na análise quantitativa de dados. Esta análise envolveu o emprego de técnicas estatísticas uni e multivariadas; o estudo pretendeu demonstrar ser possível estabelecer uma conversação entre três vertentes teóricas aparentemente divergentes: a primeira representada pela orientação para o mercado e sua perspectiva comportamentalista; a segunda subordinada à aferição do desempenho organizacional, com suas características essencialmente técnicas e objetivas; e a terceira, de natureza eminentemente subjetiva, obtida pela percepção externa de sucesso ou insucesso corporativo por parte de experts no setor de C\&T.

\section{A população em estudo}

O universo desta pesquisa foi composto por instituições de pesquisas tecnológicas associadas à ABIPTI. Foi tomada a decisão de utilizar a base de indicadores ABIPTI, por ser a mais completa e tradicional do setor. O número expressivo de instituições afiliadas, conforme descrito a seguir, praticamente esgota a possibilidade de haver alguma instituição nesse campo de atividades que não seja filiada à ABIPTI.

Em julho de 2010, essa entidade contava com 234 associados, de acordo com informações oficiais de seu site (ABIPTI, 2010), incluindo não apenas instituições de pesquisas tecnológicas, mas também associações de classe envolvidas com Ciência \& Tecnologia, nos âmbitos regional e nacional e, ainda, em órgãos públicos e privados de fomento às atividades de C\&T.

Os institutos de pesquisas tecnológicas, especificamente, totalizavam 140 na época da pesquisa de campo (janeiro/maio de 2010). Esse montante foi reduzido para os 96 institutos de pesquisa que se haviam inicialmente inscrito como participantes no Projeto Excelência na Pesquisa Tecnológica. Esta quantidade foi, mais uma vez, reduzida para apenas 70 institutos de pesquisas tecnológicas, públicos e privados, que enviaram efetivamente seus indicadores de desempenho no período de onze anos, compreendido entre 1995 e 2006, compondo, finalmente, a população-alvo deste trabalho.

\section{Os métodos e instrumentos de coleta de dados}

A pesquisa contou com dois métodos de coleta de dados. Para aferir o desempenho organizacional, foi efetuada uma análise de dados secundários, a partir de uma extensa consulta efetuada ao banco de dados de indicadores de desempenho do Projeto Excelência ABIPTI. Por outro lado, tanto para colher o grau de orientação para o mercado no âmbito das instituições de pesquisas 
tecnológicas, por meio de um questionário MARKOR adaptado, quanto para levantar a percepção externa de sucesso ou insucesso corporativo, mediante a utilização do Painel de Especialistas, foi utilizada a coleta de dados primários por meio de duas surveys a distância deflagradas em paralelo, as quais se deram, na prática, pela remessa de questionários autoadministráveis anexos a mensagens de emails, que continham, por sua vez, instruções detalhadas para o preenchimento do questionário e um texto persuasivo à colaboração. Isto, porém, foi feito somente após uma cuidadosa atualização de dois cadastros de respondentes em potencial. Um deles integrado pelos dirigentes das setenta instituições de pesquisas tecnológicas levadas a efeito para compor o construto orientação para o mercado e o outro cadastro composto por indivíduos conhecedores do setor de C\&T brasileiro, porém sem quaisquer vínculos com as instituições de pesquisas tecnológicas a que se procedem, para constituir o construto percepção externa de sucesso ou insucesso corporativo.

\section{Aferição do construto desempenho organizacional: indicadores de desempenho ABIPTI}

O fator desempenho organizacional foi obtido, a partir da compilação das séries históricas de indicadores de desempenho, enviadas entre 1995 e 2006 pelos institutos de pesquisas tecnológicos associados à ABIPTI, que participaram do Projeto Excelência na Pesquisa Tecnológica e especialmente disponibilizadas por aquela Associação para este trabalho. As variáveis de desempenho organizacional, cada uma representada por um indicador de desempenho estabelecido pela ABIPTI, passaram inicialmente por uma fase exploratória descritiva, em que se empreendeu um estudo estatístico, tendo como unidades de análise as setenta instituições de pesquisas tecnológicas que compuseram a população-alvo e, como unidades de observação, os indicadores de desempenho componentes da série histórica do Projeto Excelência da ABIPTI; um problema enfrentado nessa etapa foi a diversidade encontrada nos resultados das fórmulas de cálculo adotadas na composição dos indicadores de desempenho organizacional, uma vez que estes se apresentavam, alternadamente, sob forma de números absolutos, números índices e valores percentuais. Para contornar esse problema, adotou-se a sistemática de utilizar os valores padronizados dos indicadores.

Para compor o construto desempenho organizacional de cada um dos 70 institutos estudados, foram calculados os somatórios para a série histórica (1995-2006), apresentada por todos os 62 indicadores de desempenho (a série histórica de indicadores foi fornecida pela ABIPTI). A Tabela 1, a seguir, exemplifica a forma de cálculo de dois indicadores entre os 62. Os somatórios (1995-2006) de todos os indicadores compuseram a posição relativa (ranking) apresentada pelos 70 institutos no que se refere ao construto desempenho organizacional.

Tabela 1

Exemplo da Planilha de Cálculo para os Escores Finais de Desempenho Organizacional

\begin{tabular}{lccccccc}
\hline $\begin{array}{l}\text { INDICADORES / } \\
\text { INSTITUTOS }\end{array}$ & $\begin{array}{c}\text { Cód. 103 } \\
\text { valor } \\
\text { absoluto }\end{array}$ & $\begin{array}{c}\text { Cód. 104 } \\
\text { valor } \\
\text { absoluto }\end{array}$ & $\begin{array}{c}\text { Cód. 103 } \\
\text { valor } \\
\text { padronizado }\end{array}$ & $\begin{array}{c}\text { Cód. 104 } \\
\text { valor } \\
\text { padronizado }\end{array}$ & $\begin{array}{c}\text { Somatório } \\
\text { Padronizado } \\
\text { 1995-2006 }\end{array}$ \\
\hline $\begin{array}{l}\text { CCDM - Centro de } \\
\text { Caracterização e }\end{array}$ & 912,81 & 39,00 & $\mid$ & 1,9889 & $-0,6858$ & $\mid$ & $\mathbf{1 , 3 0 3 1}$ \\
$\begin{array}{l}\text { Desenvolvimento de Materiais } \\
\text { CDTN - Centro de }\end{array}$ & & & & & & & \\
$\begin{array}{l}\text { Desenvolvimento da Tecnologia } \\
\text { Nuclear }\end{array}$ & - & 14,27 & $\mid$ & - & 0,2251 & $\mid$ & $\mathbf{0 , 2 2 5 1}$ \\
$\begin{array}{l}\text { CENPES/PETROBRAS - Centro } \\
\text { de Pesquisas e Desenvolvimento } \\
\text { Leopoldo A. Miguez de Mello }\end{array}$ & 147,00 & 49,10 & $\mid$ & $-0,5355$ & 0,0133 & $\mid$ & $\mathbf{- 0 , 5 2 2 2}$ \\
\hline
\end{tabular}


Tabela 1 (continuação)

\begin{tabular}{|c|c|c|c|c|c|c|c|}
\hline $\begin{array}{l}\text { INDICADORES / } \\
\text { INSTITUTOS }\end{array}$ & $\begin{array}{l}\text { Cód. } 103 \\
\text { valor } \\
\text { absoluto }\end{array}$ & $\begin{array}{l}\text { Cód. } 104 \\
\text { valor } \\
\text { absoluto }\end{array}$ & I & $\begin{array}{l}\text { Cód. } 103 \\
\text { valor } \\
\text { padronizado }\end{array}$ & $\begin{array}{c}\text { Cód. } 104 \\
\text { valor } \\
\text { padronizado }\end{array}$ & | & $\begin{array}{l}\text { Somatório } \\
\text { Padronizado } \\
\text { 1995-2006 }\end{array}$ \\
\hline $\begin{array}{l}\text { CEPED - Centro de } \\
\text { Pesquisas e Desenvolvimento } \\
\text { do Estado da Bahia }\end{array}$ & 218,96 & 64,71 & | & $-0,2983$ & 0,4668 & | & 0,1685 \\
\hline $\begin{array}{l}\text { MPEG - Centro de } \\
\text { Caracterização e } \\
\text { Desenvolvimento de } \\
\text { Materiais }\end{array}$ & 24,05 & 383,39 & | & $-0,7068$ & 1,1977 & | & 0,4909 \\
\hline $\begin{array}{l}\text { NUTEC - Fundação Núcleo } \\
\text { de Tecnologia Industrial do } \\
\text { Ceará }\end{array}$ & 17,56 & 135,02 & | & 0,7376 & 0,3820 & | & 1,1196 \\
\hline ON - Observatório Nacional & 100,00 & - & | & $-0,5974$ & - & | & $-0,5974$ \\
\hline $\begin{array}{l}\text { TECPAR - Instituto de } \\
\text { Tecnologia do Paraná }\end{array}$ & 60,11 & 17,21 & | & $-0,6610$ & $-0,5510$ & | & $-1,212$ \\
\hline
\end{tabular}

Nota. (a) INDICADOR 103 - Captação de recursos provenientes do organismo mantenedor; (b) INDICADOR 104 Captação de recursos provenientes do faturamento total; (c) Dados fictícios, apenas para exemplificação.

Fonte: Elaborada pelos autores.

\section{Aferição do construto orientação para o mercado: a escala MARKOR adaptada}

A Escala MARKOR foi originalmente desenvolvida no idioma inglês. No presente estudo, foi utilizada uma versão ligeiramente modificada daquela empregada anteriormente por Sampaio (2000) e que sofreu ainda pequenas modificações (por exemplo, organização ao invés de empresa), para se adequar ao setor ora pesquisado. Essa escala já havia sido cuidadosamente validada para a língua portuguesa pelo próprio Sampaio (2000), em seu trabalho sobre a orientação para o mercado em empresas de varejo de confecção do Brasil. Naquela oportunidade, o autor relatou que a escala fora primeiramente convertida para o português, respeitando-se os conceitos empregados no trabalho original, por meio da técnica denominada tradução reversa (Dillon, Madden, \& Firtle, 1994), segundo a qual, primeiramente, três acadêmicos de marketing com domínio da língua inglesa traduzem, cada qual isoladamente, a escala para o português. Em seguida, essas traduções para a língua portuguesa são avaliadas, unificadas e convertidas novamente para o Inglês por outro acadêmico de marketing nativo na língua inglesa e, finalmente, dois outros acadêmicos de marketing compararam a versão resultante com a tradução efetuada diretamente da escala MARKOR original, buscando-se, assim, garantir a precisão no entendimento dos termos e significados em nosso idioma.

Anteriormente à etapa de coleta de dados, uma versão do questionário MARKOR foi adaptada de forma a se adequar aos propósitos desta pesquisa, para poder ser aplicada nos dirigentes das instituições de pesquisas tecnológicas componentes da população estudada; mas antes, aquele formulário sofreu pequenos ajustes resultantes de um pré-teste realizado em duas fases, para evitar uma eventual má interpretação por parte dos respondentes e, consequentemente, vieses nas respostas, com os respondentes, como sempre, desconhecendo que o objetivo maior da iniciativa não era exatamente obter suas informações e, sim, testar a aderência do instrumento de coleta aos objetivos da pesquisa; realizadas as modificações sugeridas pelos respondentes, assumiu-se, então, que o questionário seria compreendido pelos respondentes e poderia ser aplicado, sem maiores problemas, nas setenta instituições de pesquisas tecnológicas integrantes do universo pesquisado.

A etapa de coleta de dados, propriamente dita, se deu entre janeiro e maio de 2010; isto só foi possível, devido ao baixo custo e à agilidade do instrumento de coleta utilizado: um questionário autoadministrável, construído em Word e anexo aos e-mails enviados aos respondentes qualificados. 
Para tal, foi preciso montar previamente um cadastro composto pelos setenta respondentes-alvo, o que envolveu realizar grande quantidade de consultas aos sites das instituições pesquisadas e de efetuar centenas de telefonemas, a fim de atualizar a grafia correta dos nomes e cargos dos respondentes e de seus assessores diretos, assim como os logins corretos de seus e-mails. Como resultante desses procedimentos cuidadosos e sistemáticos, a taxa de mortalidade (não-respostas) do trabalho de campo foi praticamente zero, uma vez que se adotou a norma de nunca enviar e-mails com o questionário MARKOR anexo, sem que fossem feitos correspondentes telefonemas prévios aos destinatários, explicando os objetivos da pesquisa, pedindo-lhes autorização para o envio de e-mails e exortando-os a colaborar.

Uma vez aplicada no campo a versão do questionário MARKOR, adaptada às peculiaridades do setor estudado, foi testada ainda a confiabilidade daquele instrumento de coleta. O Alpha de Cronbach é importante indicador estatístico da fidedignidade de uma escala. A pontuação de cada item é computada, e a classificação final é definida pela soma de todas as pontuações intermediárias. O Alpha de Cronbach consiste no quadrado da correlação entre as pontuações de uma escala e o fator subjacente que essa escala se propõe a medir. Hair et al. (2005) postulam, como regra geral, que um bom valor para o Alpha seria 0,70 ou superior (obtidos com dados de uma amostra significativa), sendo aceitos, como patamares mínimos, valores iguais ou acima de 0,60 no caso de pesquisas exploratórias como a presente.

Foi calculado o Alpha de Cronbach para medir a confiabilidade interna entre as respostas dos setenta questionários MARKOR, preenchidos pelos CEOs dos institutos de pesquisas estudados; os resultados encontrados foram de 0,911 para os valores absolutos das respostas; e de 0,914 para os mesmos valores, porém, padronizados, ambos com uma significância inferior a 0,001 , o que corrobora a confiabilidade da versão da escala MARKOR adaptada ao setor estudado e utilizada nesta pesquisa.

Além disso, outro procedimento preventivo foi adotado, no sentido de referendar a validade do instrumento de coleta de dados para este construto, que é a proporção de missing values (valores perdidos ou omitidos, lacunas) encontrada nos 70 questionários MARKOR devolvidos após o preenchimento, a qual foi considerada relativamente pequena. Em apenas treze questionários havia, de um a, no máximo, seis quesitos em branco para um total de vinte quesitos por questionário. Com isto foi considerada confiável a massa de dados obtida para aferir esse construto.

\section{Aferição do fator sucesso ou insucesso corporativo: o painel de especialistas}

Simultaneamente à coleta de dados para a montagem do construto orientação para o mercado, um Painel de Especialistas no setor de Ciência \& Tecnologia foi recrutado e convidado a opinar sobre os graus, supostamente diferenciados, de sucesso ou insucesso corporativo no desempenho das atividades-fim das instituições de pesquisas tecnológicas estudadas.

No caso particular do presente estudo, o objetivo foi obter uma avaliação coerente, já que o interesse era identificar a existência de um continuum para o fator: sucesso ou insucesso corporativo, a partir do qual foi extraída a definição oficial, adotada neste artigo, apresentada a seguir:

O sucesso ou insucesso corporativo das instituições de pesquisas tecnológicas brasileiras referese ao fato de elas, independentemente da área de conhecimento em que atuem, apresentarem ao público especializado externo um desempenho perceptivelmente diferenciado (seja superior ou inferior) em suas atividades-fim, ou seja, aquelas diretamente relacionadas com o desenvolvimento de estudos que permitam a criação e o oferecimento contínuo de produtos e serviços tecnológicos, que atendam aos propósitos do governo, do mercado e da sociedade em geral.

Foi utilizada, portanto, a percepção como forma de medida, por meio da utilização da opinião agregada e do julgamento de um painel, composto por indivíduos altamente qualificados e experientes, que constituiu um grupo independente de especialistas e conhecedores do setor brasileiro de C\&T; porém sem que nenhum deles tivesse quaisquer vínculos empregatícios ou de consultoria com as instituições das pesquisas tecnológicas avaliadas neste trabalho. 
O trabalho de cadastramento de possíveis participantes e de coleta de opiniões para o Painel de Especialistas transcorreu entre janeiro e maio de 2010, simultaneamente e com procedimentos similares aos da tarefa de coleta de dados para os questionários MARKOR. Foram contatados e convidados a participarem, ao todo, 170 indivíduos, dos quais 68 responderam, o que corresponde a 40\%. A composição final do Painel de Especialistas contou com 38,2\% de executivos e técnicos de órgãos governamentais, ligados a C\&T; $25,0 \%$ de professores e pesquisadores com atuação em C\&T; $22,1 \%$ de dirigentes e técnicos de associações de classe ligadas a C\&T e 14,7\% de executivos e técnicos da área de $\mathrm{P} \& \mathrm{D}$ de empresas.

O recrutamento e o convite à participação dos potenciais respondentes foram feitos, sucessivamente, a partir de indicações dos respondentes que os antecediam, até se esgotar o prazo estabelecido para essa etapa de trabalho de campo, sem maiores preocupações quanto ao cumprimento de quotas.

Em termos operacionais, na construção do formulário de coleta de dados para o Painel de Especialistas, as instituições de pesquisas tecnológicas associadas à ABIPTI foram listadas em ordem alfabética; pediu-se aos respondentes que opinassem subjetivamente, com base apenas em suas experiências pregressas e conhecimentos sobre o setor brasileito de $\mathrm{C} \& \mathrm{~T}$, de que maneira percebiam, em termos da imagem de sucesso ou de insucesso corporativo que estas instituições transmitiam à sociedade, utilizando para tal uma escala Likert de cinco pontos, cujas opções eram: muito bemsucedida; bem-sucedida; nem bem-sucedida, nem malsucedida e muito malsucedida.

Foi permitido aos participantes do Painel de Especialistas que deixassem de opinar sobre aquelas instituições que julgassem não conhecer o suficiente para avaliar o sucesso ou o insucesso no desempenho de suas atividades-fim. Em consequência, a maior parte dos participantes $(25,0 \%)$ opinou sobre uma quantidade que variava de 21 a 30 instituições de pesquisas, seguida de perto por $22,1 \%$ dos participantes, que opinou sobre a faixa que ia de 31 a 40 instituições e por $11,8 \%$, opinando na faixa entre 31 e 40 instituições; a maior parte, portanto, 58,9\% dos experts consultados (40 em 68) emitiu opiniões a respeito de até 40 instituições, o que foi considerado razoável para a validação do emprego do instrumento de coleta Painel de Especialistas para aferir o construto percepção externa de sucesso ou insucesso corporativo.

Uma análise mais objetiva ainda foi efetuada, em seguida, sobre as respostas do Painel de Especialistas, com o objetivo de referendar a validade de seu emprego nesta pesquisa, que foi a realização de testes de correlação por postos de Spearman entre os somatórios dos escores dos 68 juízes para as 70 instituições de pesquisas estudadas. Os resultados desses testes validaram o acerto da utilização do Painel de Especialistas, apesar das eventuais omissões de opinião a respeito de determinados institutos por parte de vários especialistas consultados.

O raciocínio subjacente é que, caso as opiniões fornecidas individualmente por cada um dos raters, avaliadores, não se devessem ao acaso, ou seja, se os escores de sucesso ou insucesso corporativo, atribuídos a cada instituição avaliada refletissem, de fato, a percepção de cada especialista consultado a respeito de uma imagem diferenciada oferecida por cada instituição quanto a esse fator, correlações positivas associadas a níveis de significância estatística adequados seriam esperadas. Em outras palavras, a consistência das opiniões do conjunto de julgadores independentes proporcionaria a confiabilidade necessária para se aceitar tal forma de aferição para o continuum sucesso ou insucesso corporativo do conjunto de instituições pesquisadas, e isto ocorreu, de fato, na maioria dos casos.

Foram calculados os coeficientes de correlação por postos de Spearman entre os somatórios dos escores de sucesso ou insucesso corporativo, atribuídos às 70 instituições de pesquisas estudadas por cada participante do Painel. O resultado obtido no somatório agregado dos mesmos escores computados para os 67 juízes remanescentes. Ou seja, isolou-se a soma dos escores individuais atribuídos por cada participante e comparou-se com o somatório agregado dos escores dos outros 67 participantes, excetuando os escores daquele participante em particular. 
Os resultados corroboraram o acerto do emprego do Painel de Especialistas como instrumento de coleta de dados e de aferição do construto percepção externa de sucesso ou insucesso corporativo nesta pesquisa. Em 73,5\%, ou seja, em 50 entre 68 casos, os testes de correlação por postos de Spearman, entre os somatórios dos escores de cada julgador individual, em relação aos julgamentos agregados dos 67 juízes remanescentes, apresentaram correlações positivas, que iam de 0,890 a 0,352, todas associadas a significâncias estatísticas expressivas menores que 0,01. Em aproximadamente $7,4 \%$ dos casos (cinco em 68), as correlações positivas apresentadas iam de 0,766 a 0,315 , todas com significâncias estatísticas, ainda expressivas, inferiores a 0,05 . Por outro lado, os seis casos em 68 $(8,8 \%$, aproximadamente), que apresentaram coeficientes de correlação por postos entre 0,698 e 0,349 , o fizeram com significâncias estatísticas associadas, ainda não desprezíveis, em se tratando de uma pesquisa relacionada ao campo das ciências sociais aplicadas, acima de 0,05 e abaixo de 0,09. Finalmente, com significâncias acima de $10 \%$, outros quatro casos apresentaram coeficientes de correlação por postos positivos, porém baixos, entre 0,338 e 0,202 e, em apenas dois outros casos, as correlações apuradas foram negativas.

\section{Operacionalização dos testes de hipóteses e de hipóteses derivadas}

Os procedimentos de operacionalização dos testes de hipóteses e de hipóteses derivadas da pesquisa envolveram testes com as estatísticas Coeficiente de Concordância de Kendall e Coeficiente de Correlação por Postos de Spearman, sempre utilizando as posições relativas (rankings) apresentadas pelos 70 institutos estudados em relação aos três construtos: desempenho organizacional, orientação para o mercado e percepção externa de sucesso ou insucesso: o construto desempenho organizacional foi constituído pelo somatório (de 1995 a 2006) dos valores padronizados dos 62 indicadores de desempenho ABIPTI obtidos pelas 70 instituições analisadas.

\section{Os Achados da Pesquisa}

Os achados da pesquisa, após a realização dos respectivos procedimentos operacionais e cálculos estatísticos, comprovaram plenamente as quatro hipóteses e as três hipóteses derivadas de trabalho formuladas, logrando responder positivamente às indagações básicas que nortearam a realização deste trabalho.

Os testes estatísticos efetuados nas hipóteses: H1, H2, H3, H4 e nas hipóteses derivadas: H2a, $\mathrm{H} 2 \mathrm{~b}$ e H2c conseguiram validá-las integralmente no nível de significância estatística pretendido. Os valores de teste, superiores a 0,75 encontrados em: $\mathrm{H} 1, \mathrm{H} 3$ e $\mathrm{H} 4$, situaram-se em patamares relativamente mais elevados do que seus correspondentes mais modestos, porém ainda positivos (acima de 0,46) no tocante a: H2, H2a, H2b e H2c.

No âmbito prático, os achados empíricos, resumidos na Tabela 2, corroboraram amplamente as principais conclusões a que também já haviam chegado inúmeros artigos acadêmicos e relatórios de pesquisas internacionais sobre o tema orientação para o mercado e reportando a sua relação positiva e direta com a presença de bons indicadores de desempenho organizacional em organizações que tenham, ou não, a lucratividade como a principal meta institucional. Conseguiu-se provar, portanto, no presente trabalho, com razoável base de fundamentação estatística, que existe forte convergência entre os rankings construídos, a partir dos somatórios de indicadores objetivos e subjetivos de desempenho organizacional, de orientação para o mercado e de percepção externa de sucesso ou insucesso corporativo coletados nas organizações integrantes do universo pesquisado. 
Tabela 2

Resumo dos Testes de Hipóteses e de Hipóteses Derivadas da Pesquisa

\begin{tabular}{|c|c|c|c|c|}
\hline $\begin{array}{c}\text { Hipóteses e } \\
\text { Hipóteses Derivadas }\end{array}$ & Tipo do Teste & $\begin{array}{c}\text { Valor } \\
\text { Encontrado }\end{array}$ & $\begin{array}{c}\text { Significativo? } \\
\text { Sim / Não }\end{array}$ & Resultado \\
\hline H1 & $W$ de Kendall & 0,972 & $\begin{array}{l}0,000 \\
\text { Sim }\end{array}$ & $\begin{array}{l}\text { Hipótese não } \\
\text { rejeitada }\end{array}$ \\
\hline H2 & $p$ de Spearman & 0,600 & $\begin{array}{l}0,000 \\
\text { Sim }\end{array}$ & $\begin{array}{l}\text { Hipótese não } \\
\text { rejeitada }\end{array}$ \\
\hline H2a & $p$ de Spearman & 0,556 & $\begin{array}{l}0,000 \\
\text { Sim }\end{array}$ & $\begin{array}{l}\text { Hipótese Derivada } \\
\text { não rejeitada }\end{array}$ \\
\hline $\mathbf{H} 2 \mathrm{~b}$ & $p$ de Spearman & 0,539 & $\begin{array}{c}0,000 \\
\text { Sim }\end{array}$ & $\begin{array}{l}\text { Hipótese Derivada } \\
\text { não rejeitada }\end{array}$ \\
\hline H2c & $p$ de Spearman & 0,464 & $\begin{array}{c}0,000 \\
\text { Sim }\end{array}$ & $\begin{array}{l}\text { Hipótese Derivada } \\
\text { não rejeitada }\end{array}$ \\
\hline $\mathbf{H 3}$ & $p$ de Spearman & 0,811 & $\begin{array}{l}0,000 \\
\text { Sim }\end{array}$ & $\begin{array}{l}\text { Hipótese não } \\
\text { rejeitada }\end{array}$ \\
\hline H4 & $p$ de Spearman & 0,751 & $\begin{array}{c}0,000 \\
\text { Sim }\end{array}$ & $\begin{array}{l}\text { Hipótese não } \\
\text { rejeitada }\end{array}$ \\
\hline
\end{tabular}

Nota. Fonte: Dados da pesquisa. Processamento efetuado por meio do Pacote Estatístico SPSS, Versão 13.0 para Windows.

\section{Limitações da Pesquisa}

Os resultados do presente trabalho encontram-se circunscritos ao âmbito do sistema brasileiro de instituições de pesquisas tecnológicas afiliadas à ABIPTI, sejam elas de origem pública ou privada e independentemente do campo do conhecimento humano em que atuem; quaisquer extrapolações da metodologia e dos resultados obtidos no presente estudo para outros setores de atividade econômica deverão ser feitos com extrema cautela. Além disso, este estudo encontra-se limitado pela escolha das variáveis constituintes dos construtos testados e também pelos procedimentos de operacionalização nelas levados a efeito.

\section{Considerações Finais}

As organizações bem-sucedidas de hoje - sejam elas grandes ou pequenas, com ou sem fins lucrativos e atuando em mercados regionais, nacionais ou globais - precisam ter em comum um forte foco no cliente e um compromisso com a filosofia de marketing. A meta do marketing é construir e administrar relacionamentos lucrativos (não, necessariamente, no stricto sensu) e de longo prazo com a clientela atual e potencial. O marketing procura atrair novos clientes para as organizações, prometendo entregar-lhes um valor superior, bem como preservar os clientes atuais, proporcionandolhes a satisfação almejada, uma vez que opera em um ambiente globalizado e dinâmico, que pode rapidamente tornar obsoletas as estratégias vencedoras de ontem. Em suma, para serem bemsucedidas, as organizações, de qualquer tipo, precisam estar sempre fortemente orientadas para os seus mercados (Kotler \& Armstrong, 2007). 
Este trabalho apresentou uma rara incursão teórica, no Brasil e no mundo, no campo dos estudos sobre o tema orientação para o mercado, por ter tido como foco de sua investigação empírica um setor composto por organizações que não visam necessariamente ao lucro, do qual faz parte o segmento especificamente estudado, o de instituições de pesquisas tecnológicas brasileiras atuantes, indistintamente, em praticamente todos os campos do conhecimento humano, cujas características e comportamentos (principalmente, o comportamento competitivo) ainda são historicamente muito pouco conhecidos.

A motivação central, portanto, que deflagrou a realização desta pesquisa foi a de esclarecer se o conceito de orientação para o mercado, plenamente aceito e tradicionalmente enraizado no ambiente empresarial, também se faria presente em setor de atividades no qual o interesse pela maximização de lucros não constitui, necessariamente, uma prioridade estratégica, muito pelo contrário e, consequentemente, verificar se essa eventual orientação para o mercado, caso existente, estaria positivamente relacionada com uma constante busca pela melhoria no desempenho organizacional, expresso por um conjunto de indicadores apresentados pelas instituições integrantes do setor estudado.

Mais ainda, se a eventual performance diferenciada apresentada pelas diversas instituições desse setor em termos de orientação para o mercado e de desempenho organizacional seria perceptível por um conjunto de especialistas no setor brasileiro de C\&T, que classificariam aquelas instituições em um continuum de sucesso ou insucesso corporativo no desempenho de suas atividades-fim, isto é, diretamente relacionadas com a capacidade daquelas organizações em desenvolver estudos concretos que permitam a criação e o oferecimento contínuo de produtos e serviços tecnológicos, que atendam aos propósitos do governo, do mercado e da sociedade em geral. $\mathrm{O}$ que foi plenamente atingido, conforme estatisticamente demonstrado.

Artigo recebido em 23.02.2011. Aprovado em 17.08.2011.

\section{Referências}

Appiah-Adu, K. (1998). Market orientation and performance: empirical tests in a transition economy. Journal of Strategic Marketing, 6(1), 25-45. doi: 10.1080/096525498346685

Associação Brasileira das Instituições de Pesquisas Tecnológicas. (2010). Relação de associados. Recuperado em 26 julho, 2010, de http://www.abipti.org.br/Site/associados.html

Avlonitis, G. J., \& Gounaris, S. P. (1997). Marketing orientation and company performance. Industrial Marketing Management, 26(5), 385-402. doi: 10.1016/S0019-8501(96)00121-6

Baker, W. E., \& Sinkula, J. M. (1999). The synergistic effect of market orientation and learning orientation on organizational performance. Journal of the Academy of Marketing Science, 27(4), 411-427. doi: 10.1177/0092070399274002

Becker, J., \& Homburg, C. (1999). Market-oriented management: a systems-based perspective. Journal of Market-Focused Management, 4(1), 17-41. doi: 10.1023/A:1009804011447

Blau, J. R., \& Mckinley, W. (1979). Ideas, complexity and innovation. Administrative Science Quarterly, 24(2), 200-219. doi 0001-8392/79/2402-0200\$00.75

Caruana, A., Ramaseshan, B., \& Ewing, M. (1996). Market orientation and performance: a study of Australasian universities [Working Paper $N^{\circ}$ 9603]. Curtin University of Technology, Perth, Western Australia. 
Cervera, A., Mollá, A., \& Sánchez, M. (2001). Antecedents and consequences of market orientation in public organisations. European Journal of Marketing, 35(11-12), 1259-1286. doi: 10.1108/EUM0000000006476

Chandler, A. D. (1977). The visible hand. Cambridge, MA: The Belknap Press of Harvard University Press.

Didonet, S. R. (2007). Orientação para o mercado e eficiência: aplicações ao segmento supermercadista (Tese de doutorado). Universidade Federal de Minas Gerais, Belo Horizonte, MG, Brasil.

Dillon, W. R., Madden, T. J., \& Firtle, N. H. (1994). Marketing research in a marketing environment. St. Louis: Times Mirror.

Drucker, P. F. (1954). The practice of management. New York: Harper.

Egeren, M. V., \& O'Connor, S. (1998). Drivers of market orientation and performance in service firms. Journal of Services Marketing, 12(1), 39-58. doi: 10.1108/08876049810202357

Ferreira, A. R. (2003). Análise comparativa do prêmio qualidade do Governo Federal com outros prêmios nacionais e internacionais de qualidade (Dissertação de mestrado). Fundação Getúlio Vargas, Brasília, DF, Brasil.

Fleck, D. L. (2009). Archetypes of organizational success and failure. Brazilian Administration Review, 6(2), 78-100. doi: 10.1590/S1807-76922009000200002

Forehand, G. A. (1963). Assessments of innovative behavior: partial criteria for the assessment of executive performance. Journal of Applied Psychology, 47(3), 206-213. doi: 10.1037/h0049211

Fritz, W. (1996). Market orientation and corporate success: findings from Germany. European Journal of Marketing, 30(8), 59-74. doi: 10.1108/03090569610130106

Geisler, E., Furino, A., \& Kiresuck, T. J. (1990). Factors in the success or failure of industryuniversity cooperative research centers. Interfaces, 20(6), 99-109. doi: 10.1287/inte.20.6.99

Ghirlanda, M. P. M. (2001). O processo institucional de avaliação do Governo Federal brasileiro. Capital intelectual (Dissertação de mestrado). Universidade de Brasília, Brasília, DF, Brasil.

Gupta, A. K., Bhojwani, H. R., Koshal. R., \& Koshal, M. (2000). Managing the process of market orientation by publicy funded laboratories: the case of CSIR, India. $R \& D$ Management, 30(4), 289-296. doi: 10.1111/1467-9310.00182

Hair, J. F., Jr., Anderson, R. E., Tatham, R. L., \& Black, W. C. (2005). Análise multivariada de dados (5a ed.). Porto Alegre: Bookman.

Hippel, E. A. von (1977). Successful and failing internal ventures: an empirical analysis. Industrial Marketing Management, 6(3), 163-174. doi: 10.1016/0019-8501(77)90014-1

Horng, S. C., \& Chen, A. C. H. (1998). Market orientation of small and medium sized firms in Taiwan. Journal of Small Business Management, 26(3), 79-85.

Hourneaux, F., Jr., Correa, H. L., \& Maximiano, A. C. A. (2006, agosto). A contribuição dos prêmios de qualidade para a gestão do desempenho na Empresa. Anais do Seminário em Administração, São Paulo, SP, Brasil, 9. Recuperado em 29 outubro, 2010, de http://www.ead.fea.usp.br/Semead/9semead/resultado_semead/trabalhosPDF/255.pdf

Kohli, A. K., \& Jaworski, B. J. (1990). Market orientation: the construct, research propositions, and managerial implications. Journal of Marketing, 54(2), 1-18. doi: 10.2307/1251866 
Kohli, A. K., Jaworski, B. J., \& Kumar, A. (1993). MARKOR: a measure of market orientation. Journal of Marketing Research, 30(4), 467-471.

Kotler, P., \& Armstrong, G. (2007). Princípios de marketing (12a ed.) São Paulo: Pearson Prentice Hall.

Kumar, K., Subramanian, R., \& Yauger, C. (1997). Examining the market orientation performance relationship. Journal of Management, 24(2), 201-233. doi: 10.1177/014920639802400204

Mellahi, K., \& Wilkinson, A. (2004). Organizational failure: a critique of recent research and a proposed integrative framework. International Journal of Management Reviews, 5-6(1), 21-41. doi: $10.1111 / j .1460-8545.2004 .00095 . x$

Miller, D., \& Friesen, P. H. (1978). Archetypes of strategy formulation. Management Science, 24(9), 921-933. doi: $10.1287 / \mathrm{mnsc}$.24.9.921

Narver, J. C., \& Slater, S. F. (1990). The effect of a market orientation on business profitability. Journal of Marketing, 54(4), 20-35.

Paim, N. A. (1984). A inovação tecnológica na indústria brasileira de informática: algumas variáveis que diferenciam o comportamento inovador de fabricantes nacionais (Dissertação de mestrado). Universidade Federal do Rio de Janeiro, Rio de Janeiro, RJ, Brasil.

Pelham, A. M. (1997). Mediating influences on the relationship between market orientation and profitability in small industrial firms. Journal of Marketing Theory \& Practice, 5(3), 55-75.

Pelham, A. M., \& Wilson, D. T. (1996). A longitudinal study of the impact of market structure, firm structure, strategy, and market orientation culture on dimensions of small-firm performance. Journal of the Academy of Marketing Science, 24(1), 27-43. doi: 10.1007/BF02893935

Pitt, L., Caruana, A., \& Berthon, P. R. (1996). Market orientation and business performance: some European evidence. International Marketing Review, 13(1), 5-18.

Raju, P. S., Lonial, S., \& Gupta, Y. P. (1995). Market orientation and the performance in the hospital industry. Journal of Health Care Marketing, 15(4), 34-41.

Sampaio, C. H. (2000). Relação entre orientação para o mercado e desempenho empresarial em empresas de varejo de vestuário do Brasil (Tese de doutorado). Universidade Federal do Rio Grande do Sul, Porto Alegre, RS, Brasil.

Slater, S. F., \& Narver, J. C. (1994). Does competitive environment moderate the market orientationperformance relationship? Journal of Marketing, 58(1), 46-55. doi: 10.2307/1252250

Souder, W. E. (1969). The validity of subjective probability of success forecasts by R\&D projects managers. IEEE Transactions on Engineering Management, 16(1), 35-49.

Souza, W. H., \& Sbragia, R. (2002). Institutos tecnológicos industriais no Brasil: desafios e oportunidades contemporâneas. Um estudo sobre oito institutos de pesquisas tecnológicas industriais governamentais. Brasília: ABIPTI.

Tersine, R. I., \& Riggs, W. (1976). The delphi technique: a long-range planning tool. Business Horizons, 19(2), 51-56. doi: 10.1016/0007-6813(76)90081-1

Venkatraman, N., \& Ramanujam, V. (1986). Measurement of business performance in strategy research: a comparison of approaches. Academy of Management Review, 11(4), 801-814.

Whetten, D. (1980). Organizational decline: a neglected topic in organizational science. Academy of Management Review, 5(4), 577-588. doi: 10.2307/257463 
Whetten, D. (1987). Organizational growth and decline processes. Annual Review of Sociology, 13(1), 335-358. doi: 10.1146/annurev.so.13.080187.002003

Wood, V. R., Bhuian, S., \& Kiecker, P. (2000). Market orientation and organizational performance in not-for-profit hospitals. Journal of Business Research, 48(3), 213-226. doi: 10.1016/S01482963(98)00086-1 